\section{(6) OPEN ACCESS}

\title{
The distribution of new HIV infections by mode of exposure in Morocco
}

\author{
Ghina R Mumtaz, ${ }^{1}$ Silva P Kouyoumjian, ${ }^{1}$ Nahla Hilmi, ${ }^{1}$ Ahmed Zidouh, ${ }^{2}$ \\ Houssine El Rhilani, ${ }^{3}$ Kamal Alami, ${ }^{3}$ Aziza Bennani, ${ }^{4}$ Eleanor Gouws, ${ }^{5}$ \\ Peter Denis Ghys, ${ }^{5}$ Laith J Abu-Raddad ${ }^{1,6,7}$
}

\begin{abstract}
Arabic Abstract translation
- Additional material is published online only. To view please visit the journal online (http://dx.doi.org/10.1136/ sextrans-2012-050844).
\end{abstract}

For numbered affiliations see end of article.

\section{Correspondence to} Dr Laith J Abu-Raddad, Infectious Disease Epidemiology Group, Weill Cornell Medical CollegeQatar, Qatar Foundation, Education City, P.O. Box 24144, Doha, Qatar; lja2002@qatar-med.cornell.edu

This article is based on a report entitled "HIV Modes of Transmission and Analysis in Morocco" published online in August 2010 by the Infectious Disease Epidemiology Group at the Weill Cornell Medical

College in Qatar, the Morocco Ministry of Health, and the Joint United Nations Programme on HIVIAIDS (UNAIDS).

Received 24 October 2012 Revised 16 December 2012 Accepted 6 January 2013 Published Online First 14 February 2013

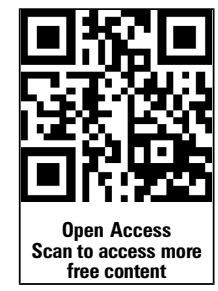

To cite: Mumtaz GR, Kouyoumjian SP, Hilmi N, et al. Sex Transm Infect 2013;89:iii49-iii56.

\section{ABSTRACT}

Objectives Building on a wealth of new empirical data, the objective of this study was to estimate the distribution of new HIV infections in Morocco by mode of exposure using the modes of transmission (MoT) mathematical model.

Methods The MoT model was implemented within a collaboration with the Morocco Ministry of Health and the Joint United Nations Programme on HIV/AIDS. The model was parameterised through a comprehensive review and synthesis of HIV and risk behaviour data in Morocco, mainly through the Middle East and North Africa HIVIAIDS Synthesis Project. Uncertainty analyses were used to assess the reliability of and uncertainty around our calculated estimates.

Results Female sex workers (FSWs), clients of FSWs, men who have sex with men (MSM) and injecting drug users (IDUs) contributed $14 \%, 24 \%, 14 \%$ and $7 \%$ of new HIV infections, respectively. Two-thirds $(67 \%)$ of new HIV infections occurred among FSWs, clients of FSWs, MSM and IDUs, or among the stable sexual partners of these populations. Casual heterosexual sex contributed $7 \%$ of HIV infections. More than half (52\%) of HIV incidence is among females, but $71 \%$ of these infections are due to an infected spouse. The vast majority of HIV infections among men (89\%) are due to high-risk behaviour. A very small HIV incidence is predicted to arise from medical injections or blood transfusions $(0.1 \%)$.

Conclusions The HIV epidemic in Morocco is driven by HIV incidence in high-risk population groups, with commercial heterosexual sex being the largest contributor to incidence. There is a need to focus HIV response more on these populations, mainly through proactive and sustainable HIV surveillance, and the expansion and increased geographical coverage of services such as condom promotion among FSWs, voluntary counselling and testing, harm reduction and treatment.

\section{INTRODUCTION}

Despite much progress on understanding HIV epidemiology globally, the Middle East and North Africa (MENA) area is the only region where knowledge of the HIV epidemic continues to be rather limited. The MENA HIV/AIDS Synthesis Project has recently provided a description of the epidemiological profile of the epidemic in MENA. ${ }^{1-5}$ Despite very limited transmission in the general population, the study identified a pattern of some established but largely emerging HIV epidemics in high-risk populations including injecting drug users (IDUs), men who have sex with men (MSM) and female sex workers (FSWs), with heterogeneity between countries on the relative role of each of these high-risk populations. ${ }^{1-4}$

HIV epidemics often show a high degree of heterogeneity in epidemic transmission and populations affected even within any given country. ${ }^{67}$ An informed response to the epidemic must accommodate the variability in HIV patterns to optimise the use of prevention and treatment resources. As almost all infections occur when an infected individual shares body fluids with an uninfected individual, prevention programmes must focus on addressing the situations in which this is happening. ${ }^{8}{ }^{9}$ Careful analysis of the patterns of HIV transmission must be conducted to inform policy decisions through a framework of 'know your epidemic; know your response. ${ }^{, 10}$

Mathematical modelling and epidemiologic synthesis are increasingly being used to inform health policy, including the design of prevention programmes and allocation of resources for HIV control. $^{1} 11 \quad 12$ This approach emphasises the importance of fine-grained analyses of the dynamics of HIV infection in a given population, and provides a systematic basis for defining national policy recommendations and strategic plans. ${ }^{12}$ The approach consolidates our knowledge of the current situation of infection transmission, and can also identify gaps in evidence and evaluate the data availability and quality about specific risk factors or modes of exposure. ${ }^{12}$

Morocco is one of the few countries in the MENA region that has made significant strides in building its HIV research capacity. Building on a wealth of newly available data, we used the modes of transmission (MoT) mathematical model to analyse the distribution of new HIV infections by mode of exposure in this country. The availability of a relatively good volume of HIV data, the admirable HIV response efforts at the national level in Morocco in comparison to other MENA countries and the presence of a highly active civil society and non-governmental organisations (NGOs) working on HIV prevention and treatment services have facilitated the MoT work in this country. This is the first MoT analysis to be conducted in the MENA region.

\section{METHODOLOGY}

Review of available data on HIV in Morocco

Data and studies on HIV epidemiology in Morocco were identified predominantly through the MENA 
HIV/AIDS Synthesis Project. ${ }^{1}{ }^{2}$ Additional data were provided through the Morocco Ministry of Health.

The MENA HIV/AIDS Epidemiology Synthesis Project included a comprehensive review and synthesis of all available biological, behavioural and contextual data about HIV in the MENA region. ${ }^{12}$ The project was conducted through a joint partnership with the World Bank, the MENA Regional Support Team of the Joint United Nations Programme on HIV/AIDS (UNAIDS) and the Eastern Mediterranean Regional Office of the WHO. The Synthesis Project covered all countries in the MENA region, including Morocco, and analysed a large number of published and unpublished data sources. The methodology, including the search strategies of each data source in the Synthesis Project, has been described in previous publications, ${ }^{12}$ and some of these details can be found in Section 1 of the online supplementary information.

The Morocco-specific data were extracted through the Synthesis Project and were complemented by additional recent data provided by the Morocco Ministry of Health and via an updated literature search of the scientific literature. Details of the data sources, studies included and the findings of the Morocco HIV epidemiological review of literature can be found in a UNAIDS report ${ }^{13}$ and a review article. ${ }^{14}$ In summary, the Morocco-specific data included: (1) articles published in the scientific literature; (2) unpublished reports of studies conducted in Morocco; (3) data provided by the Morocco Ministry of Health such as sentinel surveillance data and notified HIV/AIDS cases; and (4) HIV prevalence measures retrieved from databases of biological markers such as the US Census Bureau Database, ${ }^{15}$ among others. Studies were of different designs and sample size, and were conducted in different general and highrisk populations, and in different parts of Morocco. ${ }^{13} 14$

\section{The MoT model}

The MoT model's structure

The MoT model was used to estimate the expected short term incidence of HIV infections among the adult population stratified by mode of exposure. ${ }^{12} 1617$ The model was developed by the UNAIDS Reference Group on Estimates, Modelling and Projections. It uses input data on the current prevalence of HIV infection, the number of individuals in specific risk groups and the risk of exposure to infection within each risk group to estimate the contribution of the different risk groups in the population to HIV incidence over a period of 1 year. The model assumes the binomial function ${ }^{18}$ in terms of the number of partners and number of contacts with each partner to estimate the risk of infection for a susceptible individual in any particular risk group. Individuals are classified according to their levels of risk behaviour. The definitions of each of these population groups are summarised in table 1 . The model is implemented in a standardised template in Excel, which can be modified as necessary, where parameter values are inserted for a direct calculation of the key estimates. Further details of the model structure can be found on the UNAIDS website. ${ }^{17}$

\section{MoT model parameterisation}

The parameters of the model were chosen based primarily on available HIV epidemiologic evidence in Morocco, complemented with data from other MENA countries retrieved through the Synthesis Project, global HIV data and consensus agreement of national stakeholders for the values and ranges of plausibility for the parameters (see section 2 of the online supplementary information). In many cases, the final parameter value used was a representative or reasonable value based on a combination of the data sources mentioned above.

Table S2.1 of the online supplementary information summarises the biological parameters that were used in the MoT model, including the transmission probabilities per type of exposure act and the effect of male circumcision on the probability of HIV transmission. Note that we have incorporated the effect of sexually transmissible infections on the probability of HIV transmission per coital act implicitly. Hence the effect of sexually transmissible infections as a biological cofactor on transmission probability was not included explicitly in the model's structure. This choice reflects the findings of an extensive systematic review and meta-analysis of the probability of HIV transmission per coital act. ${ }^{19} 20$

The key demographic attributes for Morocco can be found in table S2.2 of the online supplementary information. The calculations, justifications and sources of data used for estimating population sizes of each risk group can be found in table S2.3 of the online supplementary information. The justifications and sources of data used for estimating HIV prevalence among each of the risk groups can be found in table S2.4 of the online supplementary information. Risk behaviour parameters, including the number of partnerships, number of acts of exposure and percentage of acts of exposure that are protected, are presented in tables S2.5 of the online supplementary information, along with their justifications and the sources of data used for their estimation.

Table 1 Definitions used in the model to classify individuals according to risk behaviour

\begin{tabular}{|c|c|}
\hline Risk group & Definition \\
\hline High risk populations & Individuals who are IDUs, MSM or FSWs \\
\hline Most at risk network & Individuals who engage in a risk network of IDUs, MSM or FSWs and their clients \\
\hline Individuals engaging in casual heterosexual sex (intermediate risk group) & Individuals who have had more than one sexual partner in the last year \\
\hline $\begin{array}{l}\text { Partners of IDUs, partners of clients of FSWs, female partners of MSM, } \\
\text { partners of individuals engaging in casual heterosexual sex }\end{array}$ & $\begin{array}{l}\text { Individuals who have had one sexual partner in the last year and whose partners are allocated to } \\
\text { the IDUs, clients of FSWs, MSM or casual heterosexual sex risk groups }\end{array}$ \\
\hline Individuals engaging in low-risk heterosexual sex & $\begin{array}{l}\text { Individuals who have had one heterosexual sex partner in the last year and their partners have } \\
\text { also had only one heterosexual sex partner in the last year. This group consists of individuals in } \\
\text { monogamous partnerships who are faithful to their partners and their partners are faithful to } \\
\text { them, and their partners are neither IDUs nor men MSM }\end{array}$ \\
\hline Individuals at no risk & $\begin{array}{l}\text { Individuals who have had no sexual partners in the last year. This may be a result of primary } \\
\text { abstinence (never had sexual intercourse) or secondary abstinence (had sexual intercourse in the } \\
\text { past but not currently sexually active) }\end{array}$ \\
\hline
\end{tabular}




\section{MoT uncertainty analysis}

There are uncertainties in the values of the model parameters due to a lack of studies measuring specific input parameters, limitations in the quality of studies used to provide input data, variation in estimates between studies, and/or variation in the power, representativeness and generalisability of study estimates. Therefore, we conducted an uncertainty analysis to assess the reliability of and uncertainty around our calculated estimates by using the uncertainty module of the MoT model that was developed by John Stover from the Futures Institute. ${ }^{21}$

We varied the model parameters simultaneously and randomly within a range of uncertainty for each parameter. The range of plausibility for the total annual incidence was determined by the predicted range of HIV incidence obtained from the UNAIDS SPECTRUM model ${ }^{22}$ as applied for Morocco in $2010{ }^{23}$ The median of all selected plausible outputs was then calculated and the uncertainty was described by plausibility bounds, defined as the 2.5 and 97.5 percentiles.

\section{RESULTS}

Risk group size estimates, calculated in terms of population size and the overall prevalence of each specific risk behavior, are summarised in table 2. The key predictions of the MoT model are listed in table 3, and the results of the analyses of the MoT predictions are tabulated in table 4. Table S3.1 of the online supplementary information shows the spreadsheet of the MoT model with results.

Commercial heterosexual sex (CHS) networks are the largest of the three most at-risk networks (MARNs), comprising an estimated 66859 FSWs and 668588 clients (table 2). Male same-sex sexual networks rank second, with 44573 MSM, while IDUs form the smallest high-risk population, consisting of 18500 persons (table 2). Nearly $5 \%$ of the Moroccan 1549 -year-old age group (8.2\% of males and $0.8 \%$ of females) engage in high-risk behaviour as part of at least one MARN (table 4). During any given year, $10.2 \%$ of the population (18.2\% of males and $2.3 \%$ of females) engage in intermediate to high-risk behaviour (FSWs and their clients, MSM, IDUs and people engaging in casual heterosexual sex; table 4).

An estimated 367836 persons (2.1\% of the $15-49$-year-old age group) are spouses or regular partners (predominantly women) who may be vulnerable to HIV infection as their sexual partners engage in some form of risk behaviour in MARNs (table 4). Similarly, table 2 shows an estimated 452411 spouses or regular partners $(2.5 \%$ of the $15-49$-year-old age group) who may be vulnerable, though at substantially reduced risk, because their partners engage in casual heterosexual sex.

The distribution of new HIV infections by mode of exposure is presented in table 3 and is schematised in figure 1 along with the uncertainty around the model predictions. The largest share of HIV incidence in any one risk group occurs among the low-risk heterosexual population (26\%; 95\% CI: 18-38\%), followed by clients of FSWs (24\%; 95\% CI: $13-32 \%)$ and FSWs (14\%; 95\% CI: 8-20\%). MARNs, along with the stable sexual partners of their members, predominantly drive the epidemic and contribute a total of at least $67 \%(95 \%$ CI: $55-76 \%)$ of HIV infections. Of this sum, CHS networks contribute $46 \%$ (95\% CI: $31-57 \%)$ of incident infections, followed by MSM networks (14\%; 95\% CI: 8-25\%) and IDU networks (7\%; 95\% CI: 4-13\%). The remaining one-third of HIV incidence is distributed among the rest of the population groups, including people engaged in casual heterosexual sex and their stable partners (7\%; 95\% CI: 4-11\%), the low-risk population (26\%; 95\% CI: 18-38\%), and medical injections or blood transfusions (0.11\%; 95\% CI: $0.08-0.15 \%$ ) (table 3 and figure 1). It is estimated that $71 \%$ of all HIV infections among women are due to an infected spouse (table 4) and that slightly more than half (52\%) of Morocco's HIV incidence is among females (table 3). Meanwhile, $89 \%$ of new HIV infections among men are due to engagement in some form of risk behaviour as part of a MARN (table 4).

The highest incidence rate is predicted to be among IDUs at $1.07 \%$ per person-year, followed by MSM at $1.04 \%$ and then by FSWs at $0.74 \%$ (table 3 ). Although HIV incidence rate is very small in the low-risk population at only $0.01 \%$ per personyear, the actual number of new infections is largest at 906 HIV cases per year. This is explained by the fact that this risk group comprises most of the sexually active population in Morocco. The incidence rate estimates suggest that HIV prevalence may reach, at endemic equilibrium, levels as high as roughly $13 \%$ among IDUs, 13\% among MSM and 9\% among FSWs (table 3). However, HIV prevalence in the 15-49-year-old age group of the overall population is likely to remain low and not reach a value close to $1 \%$. These projections are derived by assuming that the current incidence rates will stay at this constant level and will not increase or decrease.

Table 2 Risk group size estimate for each HIV risk group in the Morocco modes of transmission model

\begin{tabular}{|c|c|c|c|c|c|c|}
\hline \multirow{2}{*}{ Population } & \multicolumn{3}{|c|}{ Prevalence of risk } & \multicolumn{3}{|c|}{ Population size estimate } \\
\hline & Total (\%) & Male (\%) & Female (\%) & Total & Male & Female \\
\hline IDUs & 0.10 & 0.19 & 0.02 & 18500 & 16576 & 1924 \\
\hline Stable sexual partners of IDUs & 0.05 & 0.01 & 0.09 & 9250 & 962 & 8288 \\
\hline FSWs & 0.38 & - & 0.75 & 66859 & - & 66859 \\
\hline Clients of FSWs & 3.75 & 7.50 & - & 668588 & 668588 & - \\
\hline Stable sexual partners of FSWs' clients & 1.96 & - & 3.92 & 349671 & - & 349671 \\
\hline MSM & 0.25 & 0.50 & - & 44573 & 44573 & - \\
\hline Stable female partners of MSM & 0.05 & - & 0.10 & 8915 & 8915 & \\
\hline People engaged in casual heterosexual sex & 5.75 & 10.00 & 1.50 & 1025168 & 891450 & 133718 \\
\hline Stable partners of people engaged in casual heterosexual sex & 2.54 & 0.08 & 5.00 & 452411 & 6686 & 445725 \\
\hline People with low-risk heterosexual sex & 57.67 & 61.73 & 53.61 & 10282092 & 5502766 & 4779326 \\
\hline People with no-risk heterosexual sex this year & 27.50 & 20.00 & 35.00 & 4902975 & 1782900 & 3120075 \\
\hline Population at risk of medical injections & 100.00 & 100.00 & 100.00 & 17829000 & 8914500 & 8914500 \\
\hline Population at risk of blood transfusions & 0.20 & 0.20 & 0.20 & 35658 & 17829 & 17829 \\
\hline
\end{tabular}


Table 3 Key predictions of the modes of transmission model for Morocco

\begin{tabular}{|c|c|c|c|c|c|c|c|c|c|}
\hline Population & $\begin{array}{l}\text { HIV } \\
\text { prevalence } \\
(\%)\end{array}$ & $\begin{array}{l}\text { Number } \\
\text { of PLHIV }\end{array}$ & $\begin{array}{l}\text { HIV } \\
\text { incidence }\end{array}$ & $\begin{array}{l}\text { Percentage of } \\
\text { total incidence } \\
(\%)\end{array}$ & $\begin{array}{l}\text { Incidence } \\
\text { among } \\
\text { males }\end{array}$ & $\begin{array}{l}\text { Incidence } \\
\text { among } \\
\text { females }\end{array}$ & $\begin{array}{l}\text { Incidence of } \\
\text { females/ } \\
\text { total (\%) }\end{array}$ & $\begin{array}{l}\text { HIV incidence/ } \\
\text { person-year } \\
(\%)\end{array}$ & $\begin{array}{l}\text { Endemic } \\
\text { prevalence } \\
\text { (forecast) (\%)* }\end{array}$ \\
\hline IDUs & 2.00 & 370 & 197 & 5.72 & 177 & 21 & 10.4 & 1.07 & 11.34 \\
\hline Sexual partners of IDUs & 1.00 & 93 & 28 & 0.82 & 3 & 25 & 89.6 & 0.31 & 3.54 \\
\hline FSWs & 2.00 & 1337 & 497 & 14.41 & 0 & 497 & 100.0 & 0.74 & 8.19 \\
\hline Clients & 0.50 & 3343 & 821 & 23.81 & 821 & 0 & 0.0 & 0.12 & 1.45 \\
\hline Partners of clients & 0.25 & 874 & 268 & 7.78 & 0 & 268 & 100.0 & 0.08 & 0.91 \\
\hline MSM & 2.00 & 891 & 465 & 13.49 & 465 & 0 & 0.0 & 1.04 & 11.13 \\
\hline Female partners of MSM & 1.00 & 89 & 27 & 0.79 & 0 & 27 & 100.0 & 0.30 & 3.52 \\
\hline Casual heterosexual sex & 0.22 & 2255 & 82 & 2.39 & 72 & 11 & 13.0 & 0.01 & 0.10 \\
\hline $\begin{array}{l}\text { Partners of casual } \\
\text { heterosexual sex }\end{array}$ & 0.11 & 498 & 152 & 4.40 & 2 & 149 & 98.5 & 0.03 & 0.40 \\
\hline Low-risk heterosexual sex & 0.08 & 8431 & 906 & 26.29 & 101 & 805 & 88.9 & 0.01 & 0.11 \\
\hline No risk heterosexual sex & 0.03 & 1430 & 0 & 0.00 & - & - & - & - & - \\
\hline Medical injections & 0.11 & - & 3 & 0.10 & 2 & 2 & 50.0 & 0.00 & 0.00 \\
\hline Blood transfusions & 0.03 & - & 0 & 0.01 & 0 & 0 & 50.0 & 0.00 & 0.01 \\
\hline Total adult population & 0.11 & 19612 & 3446 & 100.0 & 1641 & 1805 & 52.4 & 0.02 & 0.23 \\
\hline
\end{tabular}

${ }^{*}$ Forecast assuming the current HIV incidence as predicted by the MoT model.

PLHIV, people living with HIVIAIDS; IDU, injecting drug user; FSW, female sex worker; MSM, men who have sex with men

\section{DISCUSSION}

The sizes of the high-risk populations in Morocco relative to the adult population are somewhat comparable to those in other MENA countries. ${ }^{1}$ The prevalence of injecting drug use in Morocco is about half of the average prevalence of injecting drug use in MENA countries (0.23 per 100 adult) ${ }^{1}{ }^{24}$ and substantially lower than the average prevalence globally $(0.06-$ $1.50 \%){ }^{25}$ The prevalence of sex work among women is in the intermediate to the high range compared to other MENA countries (0.1-1.0 per 100 adult women), ${ }^{1}{ }^{2}$ but lower than that in other regions (0.1-7.4 per 100 adult women). ${ }^{26}$ The prevalence of male same-sex anal sex appears to be comparable to other countries in the MENA region ${ }^{4}$ and globally (W. McFarland, personal communication,2008) $)^{27-29}$ (2-3 per 100 men).

Almost $5 \%$ of the Moroccan adult population is at elevated risk of HIV infection, being FSWs or clients of FSWs, MSM or IDUs, compared to the rest of the population. Moreover, about $5 \%$ of the adult population appears to engage in casual heterosexual sex, putting them at an intermediate risk of HIV infection. Almost $90 \%$ of the population who engages in intermediate- to high-risk behaviours are males.

FSWs and their clients, MSM and IDUs are the core drivers of the HIV epidemic in Morocco contributing, with their stable sexual partners, two-thirds of the total HIV incidence, while comprising only $6.5 \%$ of the total adult population in the country. HIV incidence among FSWs, their clients and the stable sexual partners of the clients alone contributes about half of new HIV infections every year. MSM and IDUs combined contribute just about $20 \%$ of HIV incidence every year. Despite the relatively large number of people engaged in casual heterosexual sex, the contribution of this mode of exposure to total HIV incidence is small, at about $7 \%$. Though the low-risk population contributes $26 \%$ of new HIV infections, this HIV transmission is occurring among stable discordant couples where the HIV-infected index partner is transmitting the infection to his/ her spouse. The infected index partner has probably acquired the infection in the past through engagement in high-risk behaviour as part of a MARN. Given the levels of risk behaviour in the general population and the context of HIV infection in
MENA countries, ${ }^{12}$ it is unlikely that there is or will be a substantial HIV epidemic among the general population in Morocco in the foreseeable future.

MoT analyses have been conducted in multiple countries around the world. The results show large variations between and within regions. ${ }^{30}$ For example, in sub-Saharan Africa, much of the incidence occurs among general population groups including transmissions in stable sero-discordant couples. ${ }^{30}$ In other settings where the HIV epidemics are driven largely by high-risk populations, there is variability as to which population group is dominant. For example, in the Americas, MSM are usually the leading contributors to HIV incidence, while in West Africa and Eastern Europe, sex work and injecting drug use, respectively, are the main drivers. In MENA countries other than in Morocco, an MoT has been conducted only in Iran. ${ }^{30}$ The analysis indicated that IDU networks are the main driver of the HIV epidemic in Iran, accounting for $68 \%$ of new HIV infections. $^{30}$ The large contribution of CHS networks in Morocco is therefore a key feature that is shared, based on a substantial volume of epidemiological data, only by few other MENA countries. ${ }^{1} 2424$ In a large fraction of MENA countries, injecting drug use and anal sex between males are the main drivers of much of the endemic HIV transmission. 12424

There are several reasons that may explain the rather low incidence of HIV in Morocco compared to other settings. These include the nature of sexual networks in this country in consideration of the prevailing sexually conservative socio-cultural norms ${ }^{1} 2$ and the prevention efforts implemented in Morocco by governmental actors and NGOs, particularly in CHS networks. Male circumcision, shown to have about $60 \%$ efficacy against HIV acquisition among men in three randomised clinical trials, ${ }^{31-33}$ is another key reason. Using the MoT model, we estimated that male circumcision, which is universal in Morocco, has prevented about $32 \%$ of HIV infections in the population (results not shown). The current HIV incidence among clients of FSWs is only $41 \%$ of the incidence that would have occurred if male circumcision was not practiced in Morocco. Male circumcision is likely to be one of the reasons behind the relatively low intensity of HIV transmission in CHS networks despite 
Table 4 Results of the analyses of the findings of the modes of transmission model for Morocco

\begin{tabular}{|c|c|}
\hline Finding & Proportion \\
\hline \multicolumn{2}{|l|}{ Infections originating from: } \\
\hline CHS networks & $46.0 \%$ \\
\hline MSM networks & $14.3 \%$ \\
\hline IDU networks & $6.5 \%$ \\
\hline Three most at risk networks (CHS, MSM, IDUs) & $66.8 \%$ \\
\hline Casual heterosexual sex networks & $6.8 \%$ \\
\hline Discordant partnerships in the low-risk general population & $26.3 \%$ \\
\hline Parenteral transmissions other than injecting drug use & $0.1 \%$ \\
\hline \multicolumn{2}{|l|}{ Infections among: } \\
\hline Women due to an infected spouse & $70.7 \%$ \\
\hline Men due to high-risk behaviour (CHS, MSM, IDUs) & $89.1 \%$ \\
\hline Partners of high-risk individuals & $13.8 \%$ \\
\hline \multicolumn{2}{|l|}{ Population engaged in high-risk behaviour (CHS, MSM, IDUs): } \\
\hline Males & $8.2 \%$ \\
\hline Females & $0.8 \%$ \\
\hline Adult population & $4.5 \%$ \\
\hline $\begin{array}{l}\text { Fraction of males among population engaged in high-risk } \\
\text { behaviour }\end{array}$ & $91.4 \%$ \\
\hline $\begin{array}{l}\text { Fraction of females among population engaged in high-risk } \\
\text { behaviour }\end{array}$ & $8.6 \%$ \\
\hline \multicolumn{2}{|l|}{$\begin{array}{l}\text { Population engaged in high-risk behaviour (CHS, MSM, IDUs) or casual } \\
\text { heterosexual sex: }\end{array}$} \\
\hline Males & $18.2 \%$ \\
\hline Females & $2.3 \%$ \\
\hline Population & $10.2 \%$ \\
\hline $\begin{array}{l}\text { Fraction of males among people engaged in high-risk behaviour } \\
\text { or casual heterosexual sex }\end{array}$ & $88.9 \%$ \\
\hline $\begin{array}{l}\text { Fraction of females among people engaged in high-risk } \\
\text { behaviour or casual heterosexual sex }\end{array}$ & $11.1 \%$ \\
\hline \multicolumn{2}{|l|}{ Partners of persons engaged in high-risk behaviour (CHS, MSM, IDUs): } \\
\hline Number & 367836 \\
\hline Fraction of adult population & $2.1 \%$ \\
\hline \multicolumn{2}{|c|}{$\begin{array}{l}\text { Partners of persons engaged in high-risk behaviour (CHS, MSM, IDUs) or casual } \\
\text { heterosexual sex: }\end{array}$} \\
\hline Number & 820247 \\
\hline Fraction of adult population & $4.6 \%$ \\
\hline
\end{tabular}

prevailing high-risk behaviours. It is also, by its differential protective effects that favour males, one of the reasons that HIV incidence among women is about equal to that of men, even though about $90 \%$ of the high-risk behaviour in Morocco is practiced by men.

The MoT model predictions show that men and women contribute nearly equally to HIV incidence, in agreement with the distribution among notified cases (see table S4.1 of the online supplementary information). However, about $70 \%$ of women who become infected with HIV are predicted to acquire the infection from their regular sexual partners, highlighting the vulnerability of women in sero-discordant partnerships. This pattern is also seen in the rest of the MENA countries, where the vast majority of HIV infections among women are due to acquisitions from an infected spouse. ${ }^{1}$

One prediction of the MoT model appears to contradict the distribution of notified HIV/AIDS cases by risk factor (shown in table S4.1 of the online supplementary information). Among notified cases, $63 \%$ of women report multiple sexual partners as the risk factor (these women are largely believed to be FSWs) versus only $25 \%$ who report acquiring the infection from an infected husband. Apart from for blood donors and military recruits, there is virtually no mandatory or routine HIV testing in Morocco. Voluntary counselling and testing (VCT) coverage continues to be limited in Morocco, but FSWs are by far the leading beneficiaries of VCT services (about 25\% of VCT attendees are FSWs). ${ }^{34}$ This probably explains the higher diagnosis rate among FSWs, leading to a higher contribution among notified cases.

Parenteral HIV transmissions besides injecting drug use, such as through medical injections or blood transfusions, are predicted to be rare thanks to universal blood screening and adequate precautions in the use of medical injections. The rather low prevalence hepatitis $\mathrm{C}$ virus in the general population (see table S4.2 of the online supplementary information), which is a proxy for HIV parenteral transmission, ${ }^{35} 36$ also suggests that this mode of exposure is unlikely to be a major concern for HIV transmission in Morocco.

The limitations in our study arise from the limitations of the MoT model structure and, more importantly, limitations in the data input used to parameterise the model. The MoT model does not incorporate overlapping risk factors and calculates HIV incidence over the short-term (only 1 year). It cannot adequately capture the secondary HIV transmission effects arising from the onward transmission and percolation of HIV infection in the population. It also assumes an average risk behaviour rather than a distribution of risk behaviour within each risk group. It does not account for exogenous HIV exposures (linked to travel abroad), which tend to contribute a considerable fraction of HIV incidence in the MENA region. ${ }^{1}$ However, less than 2\% of HIV notified cases identified in Morocco are among Moroccans who live outside of Morocco or who have lived outside Morocco. ${ }^{34}$ Finally, a key limitation of this model in relation to Morocco specifically is that it is applied at the national rather than regional or local levels, and therefore the model is not optimised for addressing the heterogeneity of HIV transmission in this country. The ability to
Figure 1 Distribution of new HIV infections by mode of exposure including the uncertainty in the modes of transmission model predictions for Morocco. Access the article online to view this figure in colour.

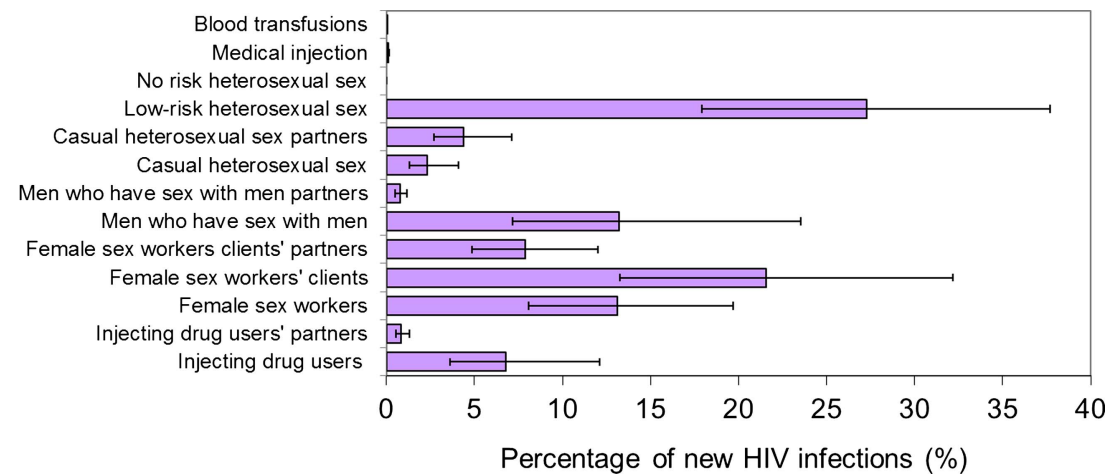


expand the application of this model at the provincial or city level in the future remains contingent on the availability of sufficient data to parameterise the model at the regional or local level.

The uncertainty analysis conducted on our model predictions suggest that there are considerable uncertainties in the quantitative predictions of our model due to the limitations in the data input used to parameterise the model. Morocco is one of the leading MENA countries in terms of the availability of HIV-related scientific evidence. Still, the limitations in our knowledge of the model parameters, due to a lack of scientific data or the limited quality or representativeness of studies, hinder our ability to make quantitatively concrete estimates. There are multiple limitations in HIV prevalence measures, risk group size estimates, and sexual and injecting risk behaviour measures. Given the uncertainties around the model parameters, we cannot make definitive statements on the size and nature of HIV epidemic transmission in Morocco.

Multiple sources of evidence were used to arrive at the model parameter values in order to have more confidence in the model data input. The overall picture emerging from our analysis, that of MARNs driving the HIV epidemic in Morocco, is likely to be robust against data input uncertainties. Evidence delineated in a review of HIV epidemiological data in Morocco is consistent and substantiate the findings of the MoT model. ${ }^{13}$ Our model findings for the year 2010 also agree well with the findings of the SPECTRUM model for Morocco for the year 2009. ${ }^{37}$ The range of HIV incidence levels predicted by the MoT model are comparable to those predicted by the SPECTRUM model (2512-4378 in the MoT model vs 1200-5800 in SPECTRUM). However, the MoT model predicted a somewhat smaller number of people living with HIV than estimated by the SPECTRUM model (19 612-26 000 in MoT vs 19 000-34 000 in SPECTRUM). ${ }^{37}$

\section{RECOMMENDATIONS}

Scientific research efforts in Morocco should focus on filling the gaps in our knowledge of the HIV epidemic in this country. The key gap is precise, and representative data on HIV prevalence, risk group size estimates and risk behaviour measures among IDUs, MSM, FSWs and their clients are needed. There is an immediate need to conduct representative and multi-centre integrated bio-behavioural surveillance surveys studies among highrisk populations using state-of-the-art sampling methodologies for hidden and hard-to-reach populations, such as respondentdriven sampling. ${ }^{38}$ Such surveys need to be repeated every few years to monitor trends and possibly to evaluate interventions. Mapping and improved risk group size estimation of high-risk populations are also a priority for HIV research and for the quantitative assessment of HIV epidemiology and trends in Morocco. A better understanding of the different typologies of these population groups (eg, street-based vs brothel-based FSWs) is warranted. The structural and vulnerability factors that lead some women to sex work need also to be understood well. Studies on high-risk populations should have broad national coverage to address the geographic heterogeneity in HIV transmission across the country, and this heterogeneity needs to be sufficiently characterised.

A population-based sexual behaviour survey of a nationally representative sample is also warranted to establish concrete data on sexual partnership formation, the nature of partnerships, the prevalence of casual heterosexual sex, the prevalence of sex work, the prevalence of contacts with FSWs, the prevalence of injecting drug use, the prevalence of male to male sexual contacts and coital frequency within different kinds of partnerships.

Morocco has made admirable strides in responding to the HIV epidemic and is probably the Arab country with the most developed HIV surveillance system, research capacity and response. ${ }^{1}$ For example, the levels of condom use among FSWs in this country are among the highest in the MENA region. ${ }^{1}$ However, despite these efforts, the overall response remains insufficient to meet current needs. An example of the low coverage of prevention efforts is the region of Souss Massa Draa, the region most affected by HIV. In this region in 2010, there were about 62 HIV tests performed per 1000 FSWs and only 17 condoms distributed per FSW. ${ }^{39}$ The estimated antiretroviral therapy coverage was only $51 \% .{ }^{39}$ Barriers to the expansion of coverage of services, such as condom distribution among FSWs by NGOs, need to be addressed.

It is essential to focus HIV response and programming on the groups at high risk of HIV infection: FSWs and their clients, MSM and IDUs. HIV counselling, testing and treatment services need to expand aggressively among these population groups. By expanding HIV testing, HIV-infected individuals who are not aware of their infection can be identified and linked to treatment, which is provided by the Moroccan Ministry of Health. The expansion of outreach and prevention programmes among MSM is urgently needed and can be done by building on the success of these programmes among FSWs. Rapid expansion of harm reduction programmes is essential in areas where injecting drug use is an important mode of transmission, such as in the north of Morocco. HIV response must be tailored to the nature of HIV transmission across the different parts of the country, with priority given to the risk groups and geographic settings where HIV is already endemic.

\section{CONCLUSION}

Our study indicates that HIV incidence among CHS networks is the leading driver of the epidemic. The HIV epidemic in CHS networks appears to be more intense than in most other MENA countries, though it is rather stable and is not at a concentrated level at the national level. ${ }^{1-3}$ However, there is a possibility that there could be hidden HIV epidemics among IDUs and MSM in Morocco that have not yet been captured by the surveillance infrastructure in this country.

There is very limited HIV transmission in the general population, with most HIV transmissions in this part of the population likely to be linked directly to transmissions originating in MARNs. It is possible that over the next decade, the HIV epidemic in Morocco may expand among FSWs and their clients, MSM and IDUs, and we may witness concentrated HIV epidemics in some of the high-risk populations in at least parts of the country. Recent data suggest that there is a concentrated HIV epidemic among IDUs in Nador in the north of Morocco, ${ }^{40}$ and among FSWs and MSM in Agadir in the south. ${ }^{14}$ HIV counselling, testing and treatment coverage among high-risk populations is still far below what is needed and needs to be expanded. The new HIV/AIDS National Strategic Plan has fixed ambitious targets to expand VCT services across Morocco and to ensure universal access. Despite the laudable progress of HIV efforts in Morocco relative to other MENA countries, there are still large gaps in our knowledge of the epidemic. Scientific research efforts must be enhanced, in line with the research priorities, in order to facilitate an evidence-informed HIV policy. 
Key messages

- Commercial heterosexual sex networks are the leading driver of the HIV epidemic in Morocco. Sexual networks of men who have sex with men and injecting networks of injecting drug users also contribute considerably to HIV incidence.

- Most risk behaviours are practiced by men. Half of the incident HIV infections are among women, but most women acquire their infection from their infected spouses.

- HIV response in Morocco needs to focus on high-risk populations through the expansion of outreach efforts and HIV services such as HIV counselling, testing and treatment.

- HIV surveillance and scientific research efforts must be enhanced, in line with data gaps identified in this analysis, to facilitate an evidence-informed HIV policy.

\section{Author affiliations \\ ${ }^{1}$ Infectious Disease Epidemiology Group, Weill Cornell Medical College-Qatar, Cornell University, Qatar Foundation, Education City, Doha, Qatar \\ ${ }^{2}$ Free-lance Epidemiologist, National consultant, Rabat, Morocco \\ ${ }^{3}$ Joint United Nations Programme on HIV/AIDS, Morocco Country Office, Rabat, Morocco \\ ${ }^{4}$ Morocco National AIDS Programme, Ministry of Health, Rabat, Morocco \\ ${ }^{5}$ Evidence, Innovation and Policy Department, Joint United Nations Programme on HIVIAIDS, Geneva, Switzerland \\ ${ }^{6}$ Department of Public Health, Weill Cornell Medical College, Cornell University, New York, New York, USA \\ ${ }^{7}$ Vaccine and Infectious Disease Division, Fred Hutchinson Cancer Research Center, Seattle, Washington, USA}

\section{Handling editor Jackie A Cassell}

Acknowledgements The Morocco HIV Modes of Transmission (MoT) study team is grateful for all the persons who participated in the national MoT workshop that was held in Morocco, and who provided valuable input in conducting this study. We would like to acknowledge the Joint United Nations Programme on HIVIAIDS (UNAIDS) headquarters and the Moroccan country office, the Ministry of Health and National AIDS Programme in Morocco, academics, non-governmental organisations and various other key public health experts and practitioners working in this field in Morocco.

Contributors LJA-R led the process of designing and conducting this study; co-led the literature reviews and the collection, analysis and interpretation of the data; and contributed to the drafting of the manuscript. GRM co-led the literature reviews and the collection, analysis and interpretation of the data; and led the drafting of the public health report on this study. SPK led the drafting of this article. All authors contributed to conducting the research, interpreting the results and drafting the public health report and this article.

Funding The Morocco HIV Modes of Transmission (MoT) Project was funded by the Joint United Nations Programme on HIVIAIDS (UNAIDS). LA, GM, and SK are grateful for the Qatar National Research Fund for their support of this work (NPRP 4-924-3-251).

\section{Competing interests None.}

Provenance and peer review Commissioned; externally peer reviewed.

Open Access This is an Open Access article distributed in accordance with the Creative Commons Attribution Non Commercial (CC BY-NC 3.0) license, which permits others to distribute, remix, adapt, build upon this work non-commercially, and license their derivative works on different terms, provided the original work is properly cited and the use is non-commercial. See: http://creativecommons.org/ licenses/by-nc/3.0/

\section{REFERENCES}

1 Abu-Raddad L, Akala FA, Semini I, et al. Characterizing the HIVIAIDS epidemic in the Middle East and North Africa: time for strategic action. Middle East and North Africa HIVIAIDS epidemiology synthesis project. Washington, DC: The World Bank Press, 2010.

2 Abu-Raddad LJ, Hilmi N, Mumtaz G, et al. Epidemiology of HIV infection in the Middle East and North Africa. AIDS 2010;24(Suppl 2):S5-23.

3 Mumtaz G, Hilmi N, Akala FA, et al. HIV-1 molecular epidemiology evidence and transmission patterns in the Middle East and North Africa. Sex Transm Infect 2011;87:101-6.

4 Mumtaz G, Hilmi N, McFarland W, et al. Are HIV epidemics among men who have sex with men emerging in the Middle East and North Africa? A systematic review and data synthesis. PLoS Med 2011:8:e1000444.
5 Abu-Raddad L, Schiffer JT, Ashley R, et al. HSV-2 serology can be predictive of HIV epidemic potential and hidden sexual risk behavior in the Middle East and North Africa. Epidemics 2010;2:173-82.

6 Kilmarx PH. Global epidemiology of HIV. Curr Opin HIV AIDS 2009:4:240-6.

7 UNAIDS. UNAIDS 2011 world AIDS day report. 2011. http://www.unaids.org/en/ media/unaids/contentassets/documents/unaidspublication/2011/JC2216 WorldAIDSday_report_2011_en.pdf (accessed 3 Jan 2012).

8 Pisani E, Garnett GP, Grassly NC, et al. Back to basics in HIV prevention: focus on exposure. BMJ 2003:326:1384-7.

9 Ainsworth M, Teokul W. Breaking the silence: setting realistic priorities for AIDS control in less-developed countries. Lancet 2000;356:55-60.

10 Wilson D, Halperin DT. "Know your epidemic, know your response": a useful approach, if we get it right. Lancet 2008:372:423-6.

11 Garnett GP, Cousens S, Hallett TB, et al. Mathematical models in the evaluation of health programmes. Lancet [Review] 2011;378:515-25.

12 Case KK, Ghys PD, Gouws E, et al. Understanding the modes of transmission mode of new HIV infection and its use in prevention planning. Bull World Health Organ 2012 2012;90:831-8.

13 Mumtaz G, Hilmi N, Zidouh $\mathrm{A}$, et al. HIV modes of transmission analysis in Morocco. Rabat, Morocco: Kingdom of Morocco Ministry of Health and National STI/AIDS Programme, Joint United Nations Programme on HIVIAIDS, and Weill Cornell Medical College-Qatar. 2010

14 Kouyoumjian SP, Mumtaz GR, Hilmi N, et al. The epidemiology of HIV infection in Morocco: systematic review and data synthesis. Int I STD AIDS 2013. In press.

15 US Census Bureau. International database. http://www.census.gov/ipc/www/idb/ (accessed 24 Jan 2013).

16 Gouws E, White PJ, Stover J, et al. Short term estimates of adult HIV incidence by mode of transmission: Kenya and Thailand as examples. Sex Transm Infect 2006;82 (Suppl 3):iii51-5.

17 UNAIDS. Epidemiological software and tools. Incidence by modes of transmission. http://www.unaids.org/en/dataanalysis/tools/incidencebymodesoftransmission/ (accessed 30 April 2012).

18 Rottingen JA, Garnett GP. The epidemiological and control implications of HIV transmission probabilities within partnerships. Sex Transm Dis 2002;29: 818-27.

19 Boily MC, Baggaley RF, Wang L, et al. Heterosexual risk of HIV-1 infection per sexual act: systematic review and meta-analysis of observational studies. Lancet Infect Dis 2009:9:118-29.

20 Baggaley RF, Boily MC, White RG, et al. Systematic review of HIV-1 transmission probabilities in absence of antiretroviral therapy. London: Imperial College London, 2004.

21 Stover J. Uncertainty estimation for the modes of transmission model. CA, USA: Futures Institute \& UNAIDS, 2009.

22 UNAIDS. Epidemiological software and tools. Spectrum/EPP 2011. http://www.unaids. org/en/dataanalysis/datatools/spectrumepp2011/ (accessed 30 April 2012).

23 El-Rhilani H. UNAIDS spectrum model for Morocco. Rabat, Morocco: UNAIDS Office, 2010

24 Mumtaz $\mathrm{G}$, Weiss $\mathrm{H}$, Thomas $\mathrm{S}$, et al. HIV epidemics are emerging among injecting drug users in the Middle-East and North Africa: systematic review and data synthesis. 2013. Under review.

25 Mathers BM, Degenhardt L, Phillips B, et al. Global epidemiology of injecting drug use and HIV among people who inject drugs: a systematic review. Lancet 2008;372:1733-45.

26 Vandepitte J, Lyerla R, Dallabetta $G$, et al. Estimates of the number of female sex workers in different regions of the world. Sex Transm Infect 2006;82(Suppl 3):iii18-25.

27 McFarland W, Caceres CF. HIV surveillance among men who have sex with men. AIDS 2001;15(Suppl 3):S23-32.

28 Mercer $\mathrm{CH}$, Hart GJ, Johnson AM, et al. Behaviourally bisexual men as a bridge population for HIV and sexually transmitted infections? Evidence from a national probability survey. Int I STD AIDS 2009;20:87-94.

29 Caceres CF, Konda K, Segura ER, et al. Epidemiology of male same-sex behaviour and associated sexual health indicators in low- and middle-income countries: 2003-2007 estimates. Sex Transm Infect 2008;84(Suppl 1):i49-56.

30 Gouws E, Cuchi P, on behalf of the International Collaboration on Estimating HIV Incidence by Modes of Transmission. Focusing the HIV response through estimating the major modes of HIV transmission: a multi-country analysis. Sex Transm Infect 2012:88:i76-85.

31 Auvert B, Taljaard D, Lagarde E, et al. Randomized, controlled intervention trial of male circumcision for reduction of HIV infection risk: the ANRS 1265 Trial. PLoS Med 2005:2:e298.

32 Bailey RC, Moses S, Parker CB, et al. Male circumcision for HIV prevention in young men in Kisumu, Kenya: a randomised controlled trial. The Lancet 2007;369:643-56.

33 Gray RH, Kigozi G, Serwadda D, et al. Male circumcision for HIV prevention in men in Rakai, Uganda: a randomised trial. The Lancet 2007:369:657-66.

34 Morocco Ministry of Health. National database of HIV data. Rabat, Morocco: National AIDS Program, 2010

35 Vickerman P, Hickman M, May M, et al. Can hepatitis C virus prevalence be used as a measure of injection-related human immunodeficiency virus risk in 
populations of injecting drug users? An ecological analysis. Addiction 2010;105:311-8.

36 Vickerman P, Martin NK, Hickman M. Understanding the trends in HIV and hepatitis $C$ prevalence amongst injecting drug users in different settings - implications for intervention impact. Drug A/cohol Depend 2012;123:1-3.

37 UNAIDS. UNAIDS country database 2007. http://www.unaids.org/en/ CountryResponses/Countries/default.asp (accessed 24 Jan 2013).
38 Magnani R, Sabin K, Saidel T, et al. Review of sampling hard-to-reach and hidden populations for HIV surveillance. AIDS 2005;19(Suppl 2):S67-72.

39 El Kettani A, Bozicevic I, El Guezzar F, et al. Understanding the dynamics of HIV epidemics in Souss Massa Draa Region in Morocco by triangulation of surveillance and programmatic data. Unpublished manuscript 2010.

40 Ministère de la Santé au Maroc, DELM/PNLS. Situation épidémiologique du VIH/Sida et des IST au Maroc. February 2010.

Download the BMJ app from iTunes and read it everywhere.

bmj.com/ipad 